\title{
Hopping in a supercooled binary Lennard-Jones liquid
}

\author{
Thomas B. Schrøder *, Jeppe C. Dyre \\ Department of Mathematics and Physics (IMFUFA), Roskilde University, P.O. Box 260, DK-4000 Roskilde, Denmark
}

\begin{abstract}
A binary Lennard-Jones liquid has been investigated by molecular dynamics at equilibrium supercooled conditions. At the lowest temperature investigated, hopping is present in the system as indicated by a secondary peak in $4 \pi r^{2} G_{\mathrm{s}}(r, t)$, where $G_{\mathrm{s}}(r, t)$ is the van Hove self correlation function. To examine the dynamics of the system, we consider transitions between the inherent structures (local minima in the potential energy) along the trajectory. We conclude that the plateau in the mean square displacement found at lower temperatures is indeed a result of particles being trapped in local "cages", as often argued, and that the system has a single-peaked distribution of hopping-distances centered around the characteristic intermolecular distance. (c) 1998 Published by Elsevier Science B.V. All rights reserved.
\end{abstract}

\section{Introduction}

Binary systems with particles differing about $20 \%$ in size are good candidates for simple models of supercooled liquids in computer simulations [13]. In some of these systems a secondary peak in $4 \pi r^{2} G_{\mathrm{s}}(r, t)$ indicates that hopping is present $[2,3]$. Since hopping of some form is expected to play an increasingly dominant role as temperature is decreased [4,5], systems exhibiting hopping at temperatures which can be reached under equilibrium conditions in computer simulations are of interest.

In the present work we investigate the dynamics of such a system [2], using the concept of "inherent structures" $[5,6]$. In the previous work the system was studied both above and below the glass transition temperature, $T_{\mathrm{g}}$, (i.e., the system was allowed to fall out of equilibrium as it was cooled). As a result it was not clear if hopping was present in equi-

\footnotetext{
${ }^{*}$ Corresponding author. E-mail: tbs@ruc.dk.
}

librium, or if it was a result of the system falling out of equilibrium. To resolve this point, in the present work we emphasise keeping the system equilibrated at all temperatures.

\section{Method}

The system studied consists of 251 particles of type $A$ and 249 particles of type $B$. The interactions are given by the Lennard-Jones potential

$$
V_{\alpha \beta}(r)=4 \epsilon\left(\left(\frac{\sigma_{\alpha \beta}}{r}\right)^{12}-\left(\frac{\sigma_{\alpha \beta}}{r}\right)^{6}\right),
$$

where $\alpha, \beta \in\{A, B\}$. The parameters are given by $\sigma_{B B} / \sigma_{A A}=5 / 6$ and $\sigma_{A B}=\left(\sigma_{A A}+\sigma_{B B}\right) / 2$, and the masses are given by $m_{B} / m_{A}=1 / 2$. The sample was cubic with sidelength $7.28 \sigma_{A A}$ and the potential between a pair of type $\alpha$ and $\beta$ was cut and shifted at $2.5 \sigma_{\alpha \beta}$. All results are reported in units of $\epsilon, \sigma_{A A}$ and $\tau \equiv\left(m_{A} \sigma_{A A}^{2} / 48 \epsilon\right)^{1 / 2}$. Adopting argon parameters for the $A$ particles leads to $\epsilon / k_{\mathrm{B}}=120 \mathrm{~K}$, $\sigma_{A A}=3.4 \AA$, and $\tau=3 \times 10^{-13} \mathrm{~s}$. The equations 
of motion were integrated with periodic boundary conditions using the Leap-Frog algorithm [7] with a time step of $0.01 \tau$.

Three independent samples were used, each initiated by generating a random configuration followed by equilibration at a temperature, $T=5.0$. The cooling was done in a step-wise manner; at each step the velocities were scaled to achieve a total energy, $E_{0}$, after which the sample was equilibrated for a time, $t_{\mathrm{eq}}$, and data were sampled for another time period, $t_{\mathrm{eq}}$. The relaxation time, $t_{\mathrm{rel}}$, was found by fitting the self part of the intermediate scattering function for the $A$ particles, $F_{\mathrm{s} A}\left(q_{\max }, t\right)\left(q_{\max }=7.5\right.$ is the approximate position of the primary peak in the static structure factor for the $A-A$ correlation), to a stretched exponential, $A \exp \left(-\left(t / \tau_{\text {rel }}\right)^{\beta}\right)$. Table 1 shows the mean values for the achieved total energy, $E_{\text {tot }},\left(\approx E_{0}\right)$, the temperature, $T$, the pressure, $p$, the relaxation time, $t_{\text {rel }}$, and the chosen equilibration time, $t_{\mathrm{eq}}$ $\left(>100 t_{\text {rel }}\right)$. The duration of the runs $\left(t_{\text {eq }}\right)$ are sufficient for $F_{\mathrm{s} A}\left(q_{\max }, t\right)$ to effectively decay to zero (not shown). Also note, that the uncertainties in Table 1 indicate, that the data for the three independent samples are in reasonable agreement. We take these facts as an indication, that the system is in equilibrium.

The quenched configuration or "inherent structure" $[5,6]$ at time $t, \mathbf{r}_{j}^{Q}(t)$, is obtained by quenching the configuration at time $t, \mathbf{r}_{j}(t)$, with steepest descent. We define the "inherent" mean square displacement for particles of type $\alpha$ (where the summation, $j$, is over particles of type $\alpha$ and $N_{\alpha}$ is the number of these)

Table 1

Values describing the samples at the three temperatures studied; The total energy, $E_{\text {tot }}$, the temperature, $T$, the pressure, $p$, the relaxation time, $t_{\text {rel }}$, and the chosen equilibration time, $t_{\mathrm{eq}}$

\begin{tabular}{rrrcl}
\hline \multicolumn{1}{c}{$\left\langle E_{\text {tot }}\right\rangle$} & \multicolumn{1}{c}{$\langle T\rangle$} & \multicolumn{1}{c}{$\langle p\rangle$} & \multicolumn{1}{c}{$\left\langle t_{\text {rel }}\right\rangle$} & \multicolumn{1}{l}{$t_{\mathrm{eq}}$} \\
\hline-2400.5 & 0.689 & 3.345 & $1.4 \times 10^{2}$ & $2.1 \times 10^{4}$ \\
\pm 0.1 & \pm 0.001 & \pm 0.002 & $\pm 0.1 \times 10^{2}$ & \\
-2496.2 & 0.636 & 3.031 & $3.7 \times 10^{2}$ & $4.2 \times 10^{4}$ \\
\pm 0.1 & \pm 0.001 & \pm 0.002 & $\pm 0.1 \times 10^{2}$ & \\
-2595.4 & 0.591 & 2.693 & $3.5 \times 10^{3}$ & $3.5 \times 10^{5}$ \\
\pm 0.2 & \pm 0.002 & \pm 0.002 & $\pm 0.1 \times 10^{3}$ & \\
\hline
\end{tabular}

Averages are over three independent samples.

$$
\left\langle\Delta r^{2}(t)\right\rangle_{\alpha}^{Q} \equiv \frac{1}{N_{\alpha}}\left\langle\sum_{j}\left(\mathbf{r}_{j}^{Q}(t)-\mathbf{r}_{j}^{Q}(0)\right)^{2}\right\rangle .
$$

This definition is identical to $\left\langle\Delta r^{2}(t)\right\rangle_{\alpha}$ except that $\mathbf{r}_{j}$ is replaced by $\mathbf{r}_{j}^{Q}$. Similarly, we define the inherent van Hove self correlation function for particles of type $\alpha$

$G_{\mathrm{s} \alpha}^{Q}(\mathbf{r}, t) \equiv \frac{1}{N_{\alpha}}\left\langle\sum_{j} \delta\left[\mathbf{r}+\mathbf{r}_{j}^{Q}(0)-\mathbf{r}_{j}^{Q}(t)\right]\right\rangle$.

Comparing these "inherent" functions with the ordinary functions, $\left\langle\Delta r^{2}(t)\right\rangle_{\alpha}$ and $G_{\mathrm{s} \alpha}(\mathbf{r}, t)$, gives information about the effect of thermal "rattling" in local minima, since this is quenched in the inherent functions. It should be noted however, that not all the thermal noise is guaranteed to be removed in the inherent functions, since these might still contain transitions over energy barriers which are insignificant at the given temperature.

\section{Results}

In Fig. 1(a) the mean square displacement of the $B$ particles, $\left\langle\Delta r^{2}(t)\right\rangle_{B}$, is shown in a log-log plot. At short times one finds the ballistic regime with a power-law exponent of 2 , and at long times the system approaches the diffusive regime with a power-law exponent 1 . Between these regimes a plateau emerges as the temperature is decreased. This is usually explained as being due the fact that the particles are trapped in local "cages" bounded by their nearest neighbors [1,8]. This is confirmed by Fig. 1(b), where the inherent mean square displacement of the $B$ particles, $\left\langle\Delta r^{2}(t)\right\rangle_{B}^{Q}$, is shown; in this case there is no plateau, because the quenching procedure eliminates the thermal "rattling". Thus, a particle trapped in a local minima does not contribute to $\left\langle\Delta r^{2}(t)\right\rangle_{B}^{Q}$. The number of particles escaping their cages increases with time, which leads to an increasing $\left\langle\Delta r^{2}(t)\right\rangle_{B}^{Q}$ in the time range where a plateau is seen for $\left\langle\Delta r^{2}(t)\right\rangle_{B}$.

Fig. 2(a) shows $4 \pi r^{2} G_{\mathrm{s}}(r, t)$ for the $B$ particles at the lowest temperature, $T=0.591$, at $t=t^{*}$ $2 t^{*}, 4 t^{*}, 8 t^{*}$ and $16 t^{*}$, where $t^{*}=328$ is the time at which the non-Gaussian parameter for the $B$ particles $\alpha_{B}$ has its maximum [9,10], as shown in 

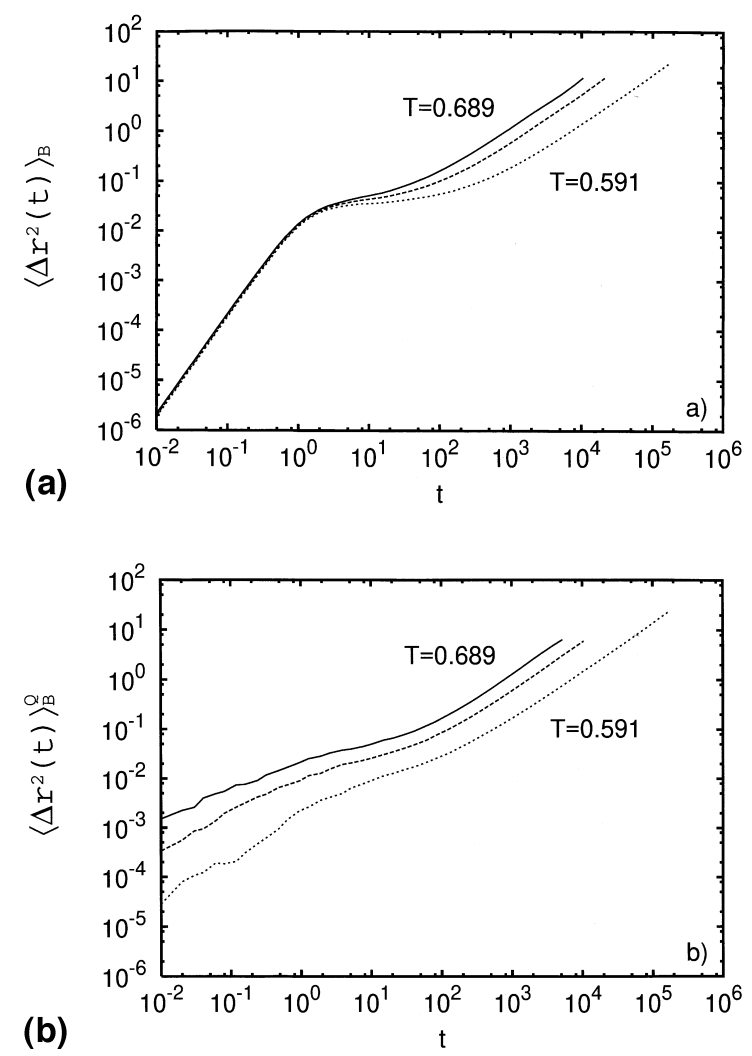

Fig. 1. (a) $\left\langle\Delta r^{2}(t)\right\rangle_{B}$ at the three temperatures in Table 1. Between the ballistic regime (short times) and the diffusive regime (long times) a plateau is seen emerging as the temperature is decreased. (b) $\left\langle\Delta r^{2}(t)\right\rangle_{B}^{Q}$ at the same temperatures as in Fig. 1(a). The plateau at intermediate times is removed by the quenching procedure.

the inset. A secondary peak is clearly seen developing, indicating hopping at this temperature. Given Fig. 2(a) one might pose the following two questions about the hopping dynamics: Is the secondary peak a sharp peak broadened by thermal noise? Are there other "hopping peaks" hidden under the primary peak? To answer these questions we look at Fig. 2(b), which shows $4 \pi r^{2} G_{\mathrm{s} B}^{Q}(r, t)$ at the same temperature and the same times as in Fig. 2(a). The hopping peak is slightly sharper, while the primary peak has moved to the left without revealing any new peaks. We conclude that the system at the given temperature has a singled-peaked distribution of hopping-distances around the characteristic intermolecular distance.
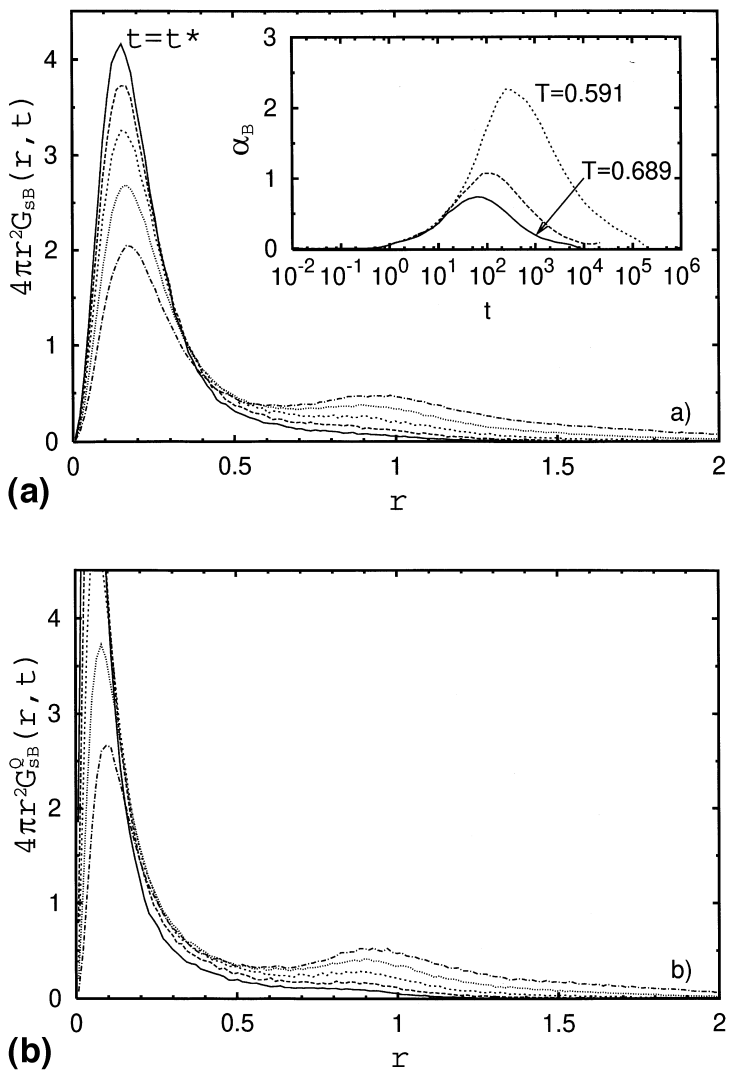

Fig. 2. (a) $4 \pi r^{2} G_{\mathrm{s} B}(\mathbf{r}, t)$ at the lowest temperature, $T=0.591$, at the times $t=t^{*}, 2 t^{*}, 4 t^{*}, 8 t^{*}$ and $16 t^{*} . t^{*}=328$ is the time at which the non-Gaussian parameter $\alpha_{B}$ has its maximum $[9,10]$, as shown in the inset. At long times a secondary peak is seen developing, indicating hopping at this temperature. (b) $4 \pi r^{2} G_{\mathrm{s} B}^{Q}(\mathbf{r}, t)$ at the same temperature and the same times as in Fig. 2(a). The hopping peak is slightly sharper than in Fig. 2(a), while the primary peak has moved to the left without revealing any new peaks.

Before we conclude, we show what we will term as a typical hopping event, judging from visual inspection of a number of these. Fig. 3 shows two quenched configurations separated $t^{*}$ in time. The configurations are shown in light and dark colors, respectively. Only particles moving more than 0.6 are shown. The figure illustrates a typical example of the kind of hopping along one-dimensional strings, which we found at the lowest temperature in this system: When one particle jumps it leaves a vacancy which a neighboring particle can jump into, and so on. Similar string-like motion has been reported Donati et al. [11]. 


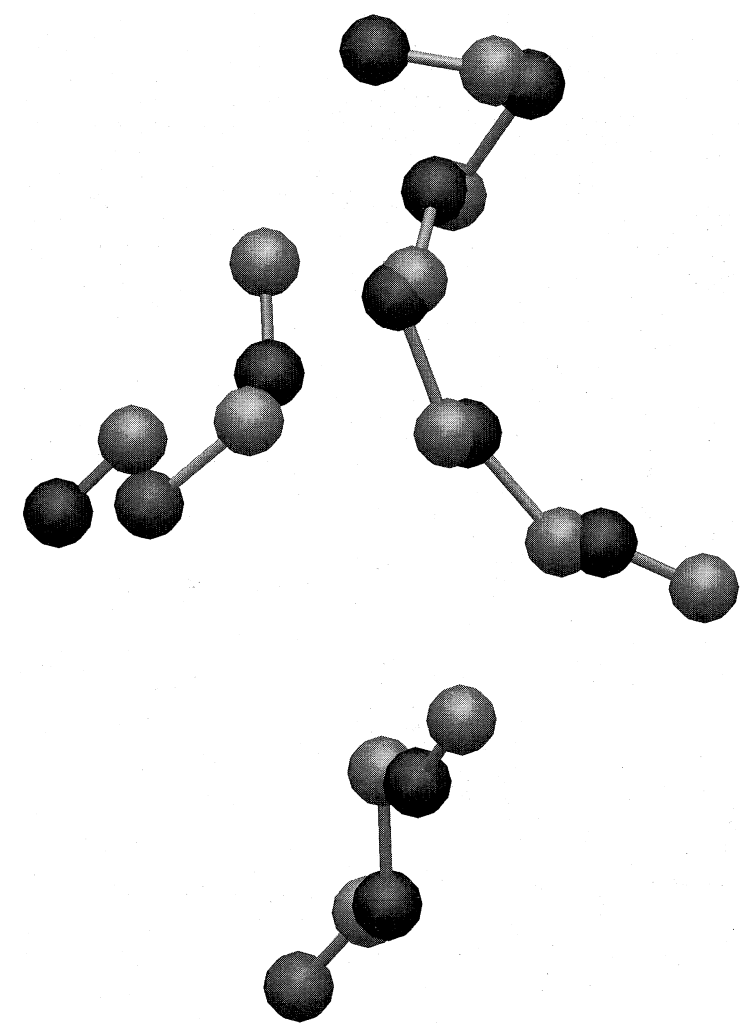

Fig. 3. A snapshot of a hopping event at $T=0.591$. The initial quenched configuration, $\mathbf{r}_{j}^{\mathcal{Q}}(t)$ (light), is connected with line segments to the final quenched configuration, $\mathbf{r}_{j}^{Q}\left(t+t^{*}\right)$ (dark). $t^{*}=328$, see Fig. 2(a). The string-like motion shown here is typical for the system at this temperature.

\section{Conclusions}

We have investigated a binary Lennard-Jones liquid under supercooled equilibrium conditions. Comparing the inherent mean square displacement for the $B$ particles, $\left\langle\Delta r^{2}(t)\right\rangle_{B}^{Q}$, with the ordinary mean square displacement for the $B$ particles, $\left\langle\Delta r^{2}(t)\right\rangle_{B}$, we have confirmed, that the plateau in $\left\langle\Delta r^{2}(t)\right\rangle_{B}$ originates from particles trapped in local "cages", i.e., local minima in the potential energy. Comparing $4 \pi r^{2} G_{\mathrm{s} B}(r, t)$ with $4 \pi r^{2} G_{\mathrm{s} B}^{Q}(r, t)$ leads us to conclude that the hopping process is characterized by a single-peaked distribution of hoppingdistances, centered around the characteristic intermolecular distance. We have thus shown, that comparing dynamic functions such as $\left\langle\Delta r^{2}(t)\right\rangle_{\alpha}$ and $G_{\mathrm{s} \alpha}(r, t)$ with the corresponding inherent functions, $\left\langle\Delta r^{2}(t)\right\rangle_{\alpha}^{Q}$ and $G_{\mathrm{s} \alpha}^{Q}(r, t)$, is a useful tool when analyzing the dynamics of a system.

\section{References}

[1] W. Kob, H.C. Andersen, Phys. Rev. E 51 (1995) 4626.

[2] G. Wahnström, Phys. Rev. A 44 (1991) 3752.

[3] J.-L. Barrat, J.-N. Roux, J.-P. Hansen, Chem. Phys. 149 (1990) 197.

[4] M. Goldstein, J. Chem. Phys. 51 (1969) 3728.

[5] F.H. Stillinger, Science 267 (1995) 1935.

[6] F.H. Stillinger, T.A. Weber, Phys. Rev. A 28 (1983) 2408.

[7] M.P. Allen, D.J. Tildesley, Computer Simulation of Liquids, Clarendon, Oxford, 1986.

[8] F. Sciortino, P. Gallo, P. Tartaglia, S.-H. Chen, Phys. Rev. E 54 (1996) 6331.

[9] T. Odagaki, Y. Hiwatari, Phys. Rev. A 43 (1991) 1103.

[10] W. Kob, C. Donati, S.J. Plimpton, P.H. Poole, S.C. Glotzer, Phys. Rev. Lett. 79 (1997) 2827.

[11] C. Donati, J.F. Douglas, W. Kob, S.J. Plimpton, P.H. Poole, S.C. Glotzer, Phys. Rev. Lett. 80 (1998) 2338. 